\title{
THE OCULAR PRESENTATION OF NEUROFIBROMATOSIS 2
}

\author{
NICOLA K. RAGGE ${ }^{1,2}$, MICHAEL E. BASER ${ }^{3}$, VINCENT M. RICCARDI ${ }^{4}$ and \\ RENA E. FALK ${ }^{2}$ \\ Los Angeles and La Crescenta, California
}

\begin{abstract}
SUMMARY
Neurofibromatosis 2 (NF2) is an inherited disorder characterised primarily by bilateral vestibular schwannomas and other central nervous system tumours. Individuals with NF2 also have early onset cortical and posterior subcapsular or capsular cataract and other ocular abnormalities, such as retinal hamartomas. Although their diagnostic significance is rarely appreciated, the ocular manifestations are often the first sign of disease. We describe 5 cases that illustrate the diverse ocular manifestations of NF2
\end{abstract}

Neurofibromatosis 2 (NF2) is a dominantly inherited disorder that predisposes the affected individual to bilateral vestibular schwannomas (VSs) and other central nervous system (CNS) tumours. The NF2 gene has been identified on chromosome band 22q12 and characterised as a putative tumour suppressor gene. ${ }^{1-3}$ In addition to the tumours, individuals with NF2 have ocular abnormalities, such as early onset cortical cataract or posterior subcapsular-capsular cataract (PSCC); and, less frequently, retinal hamartomas, or ocular motor abnormalities. ${ }^{4-7}$

NF2 occurs in mild and severe phenotypes differentiated by the presence of associated CNS tumours and age of onset. ${ }^{8-10}$ The presence of retinal hamartomas, but not cataract, may be associated with an earlier age of onset and/or a more severe phenotype. ${ }^{7,10}$ However, there is no phenotype-togenotype correlation as specific NF2 mutations have not been associated with the presence or absence of

From: ${ }^{1}$ Division of Ophthalmology, Childrens Hospital Los Angeles, and Department of Ophthalmology, USC School of Medicine, Los Angeles; ${ }^{2}$ Ahmanson Department of Paediatrics, Steven Spielberg Pediatric Research Center, Cedars-Sinai Medical Centre, UCLA School of Medicine, Los Angeles; ${ }^{3}$ Division of Neurology and Neurogenetics Laboratory, Cedars-Sinai Medical Center, UCLA School of Medicine, Los Angeles, California;

${ }^{4}$ The Neurofibromatosis Institute, La Crescenta, California, USA

Correspondence to: Miss Nicola K. Ragge, MA, MRCP, FRCOphth, Moorfields Eye Hospital, City Road, London EC1V 2PD, UK. ocular abnormalities. Preliminary evidence suggests that milder forms of NF2 may be associated with missense mutations and specific donor site mutations, while the severe phenotype may be associated with nonsense mutations, frameshift mutations and specific splice site mutations. ${ }^{11}$ However, there can be phenotypic variability with the same mutations. ${ }^{12,13}$

Due to the clinical variability and relative rarity of $\mathrm{NF} 2$, the diagnostic criteria continue to evolve. ${ }^{14}$ Awareness of the full clinical spectrum of the disease is necessary for early diagnosis and optimal management. ${ }^{14}$ The following cases illustrate some of the most characteristic ocular features of the disease.

\section{Case 1}

\section{CASE REPORTS}

A 24-year-old man with congenital esotropia and optic atrophy presented at 9 years of age with a left exotropia and decreased vision in the left eye. At 12 years he had an occipital scalp lesion removed (histology unknown), and was noted to have a single café-au-lait (CAL) macule. He subsequently developed five additional CALs. At age 18 years he underwent strabismus surgery to correct the exotropia. Seven months later he developed headaches, hearing loss, tinnitus and dizziness, diagnosed as a viral illness. An MRI scan 1 year later revealed bilateral VSs. The left VS was removed by a translabyrinthine approach at age 19 years, with subsequent left VII nerve palsy. The right VS was removed by a similar approach at age 21 years with simultaneous insertion of an auditory brainstem implant to obtain sound sensation. $\mathrm{He}$ was the firstborn child to clinically unaffected parents. On physical examination he had six CALs, slurred speech, decreased left V and VII nerve function, decreased VIII nerve function and a mildly abnormal gait.

Ocular examination revealed visual acuities of right eye 20/20, left eye 20/200, not improved with

Eye (1997) 11, 12-18 사 1997 Royal College of Ophthalmologists 
refraction. He had mild left enophthalmos with $2 \mathrm{~mm}$ left ptosis, a left exotropia of 50 prism dioptres, with restricted left extraocular movements and a $5 \mathrm{~mm}$ dilated and poorly reactive left pupil, consistent with a subtotal III nerve paresis. The right extraocular movements and pupil responses were normal. Iris hamartomas (Sakurai-Lisch nodules) were absent. $\mathrm{He}$ had bilateral mild posterior subcapsular lens opacities with a persistent hyaloid remnant posterior to the right lens. In the right fundus he had a small retinal hamartoma and a mildly dysplastic optic disc. In the left fundus he had extensive epiretinal membrane formation with macular hypoplasia and ectopia with a full not swollen optic disc, a picture possibly consistent with a combined pigment epithelial and retinal hamartoma (CPERH) (Fig. 1).

\section{Case 2}

This 15-year-old boy was the elder of two sons born to 33-year-old clinically unaffected parents. In the first few weeks of life he was found to have poor vision in his left eye and was also noted to have three cutaneous lumps and several small CALs. He was diagnosed with neurofibromatosis at age 3 years following removal of a cutaneous back lesion. At age 4 years, preschool vision screening showed decreased left visual acuity. The cause was not identified and the vision did not improve with occlusion therapy to the right eye. At age 8 years he developed a right orbital mass and a cranial MRI scan at that time additionally revealed bilateral VSs, a left hypoglossal nerve tumour and a cranial base meningioma. $\mathrm{He}$ had headaches and mild imbalance, but no tinnitus or symptomatic hearing loss, although he had mildly

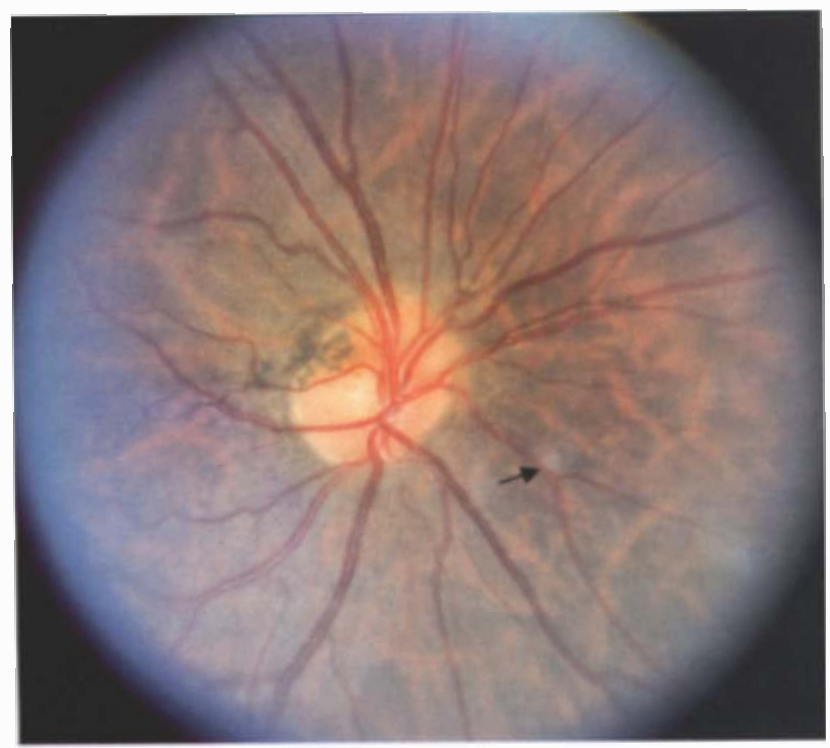

(a) increased latencies on audiometric testing. The orbital mass (a histologically confirmed schwannoma) and left VS were both resected at age 10 years. Subsequently a repeat MRI scan showed additional tumours at the cervico-medullary junction, right jugular fossa and bilateral V nerve tumours. At age 11 years, meningiomas were present in the right tuberculum sella-optic foramen region, suprasellar cistern and posterior cavernous sinus. At age 12 years he developed a left brachial plexus mass and a tumour on the right hypoglossal nerve. General physical examination revealed a large tumour on the left side of his neck with overlying pigmentation, two CALs, and two large hypopigmented macules on his limbs, several subcutaneous and cutaneous tumours on the back of his head, face, right wrist and fifth finger, right elbow and left eyebrow. He had left tongue atrophy and unsteady tandem gait.

Ocular examination showed visual acuities of 20/70 in the right eye and 7/200 in the left eye, corrected with right eye $-6.50 \mathrm{D} /+4.50 \times 87^{\circ}$, left eye $-18.50 \mathrm{D}$. On perimetry he had an enlarged blind spot on the right and a small central relative scotoma on the left. Colour vision was reduced on the right to $12 / 15$ Ishihara plates, impossible to test on the left. He had reduced right and absent left corneal sensation, and a left VII nerve paresis. He had no iris hamartomas. He had mild bilateral posterior subcapsular cataracts. There was a left afferent pupil defect. He had a small pale optic disc on the left with an extensive area of thinned retinal pigment epithelium overlying the papillomacular bundle, possibly corresponding to a combined pigment epithelial and retinal hamartoma. $\mathrm{He}$ had peripheral retinal degenerative changes,

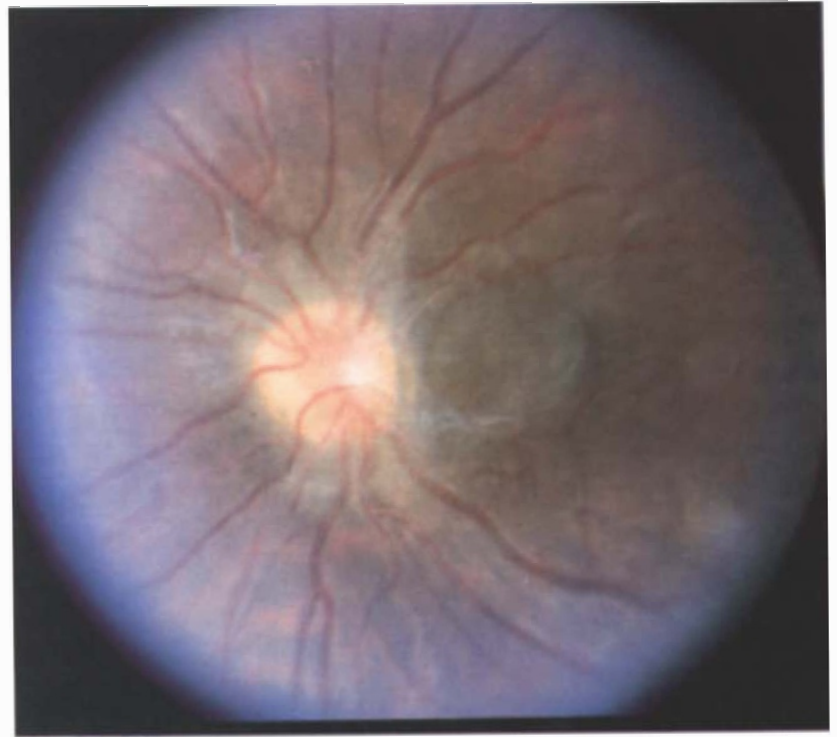

(b)

Fig. 1. Case 1. Fundus appearance. Right optic disc is mildly dysplastic (a). A small retinal hamartoma is present (arrow). The left fundus (b) shows an extensive epiretinal membrane with macular ectopia. There is also elevation of the left optic disc. 

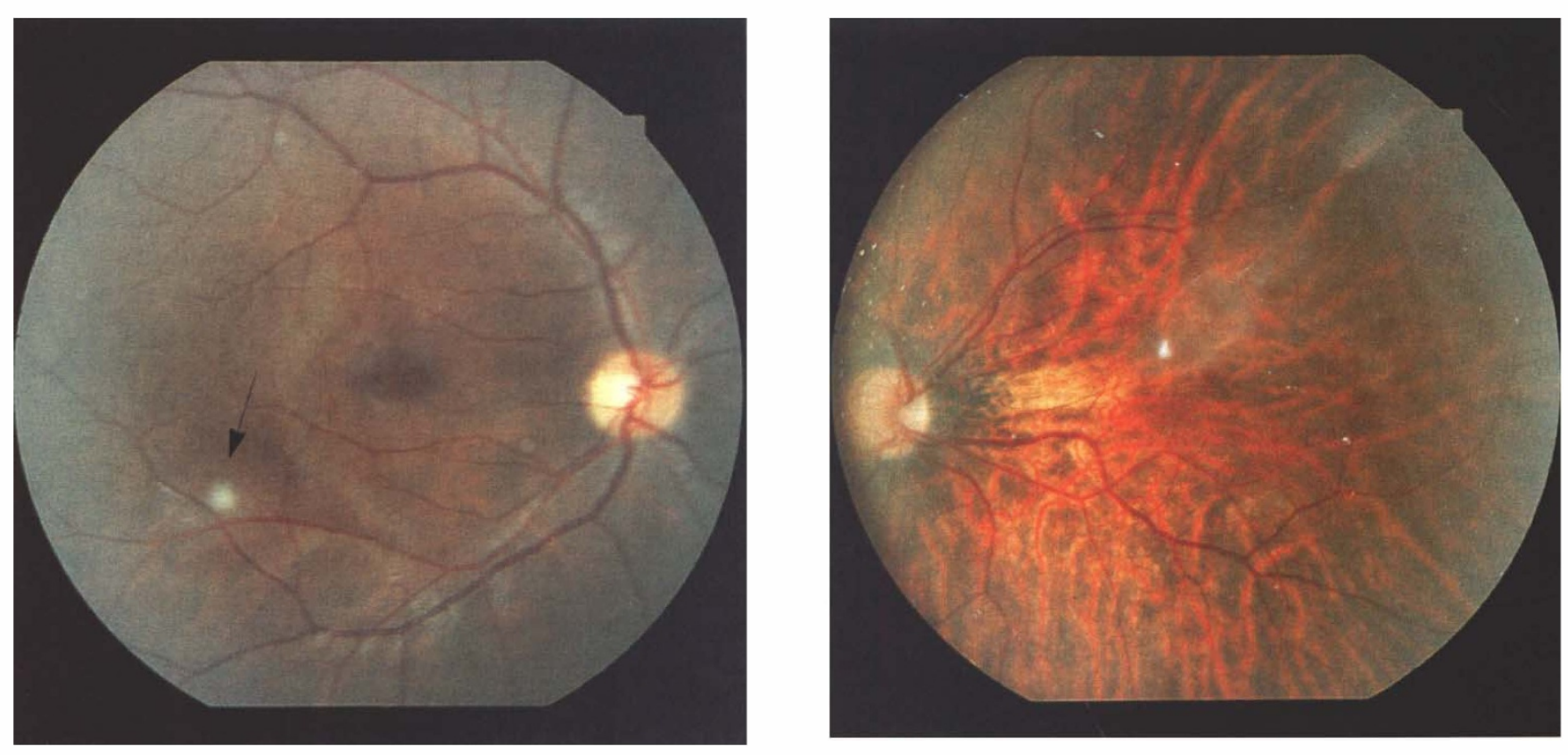

Fig. 2. Case 2. Fundus appearance of right eye (a) showing peripheral retinal hamartoma (arrow) and the left eye (b) showing pigment epithelial abnormality and epiretinal membrane in a highly myopic eye which developed a retinal detachment.

several small tears and an early retinal detachment in the left eye. In the right eye he had a small retinal hamartoma with a normal optic disc (Fig. 2).

\section{Case 3}

A 59-year-old woman with NF2 had mild blurring of vision in the left eye. Her mother, maternal aunt and maternal grandmother had a history suggestive of NF2. The patient developed a right VS at age 28 years, presenting with tinnitus and sudden onset of hearing loss. The tumour was removed at age 32 years. At age 44 years she developed a left VS treated by a left middle fossa decompression. She developed another left VS at age 47 years treated by removal and an auditory brainstem implant was inserted. Ten years later she developed a sudden VII nerve palsy due to a facial nerve tumour. At age 58 years she developed a malignant tumour in the oral cavity (histology unknown).

The patient developed left exposure keratitis and neurotropic keratitis following the removal of the left $\mathrm{VS}$, for which she received a gold weight implant in the left upper lid. She had also undergone left cataract extraction with intraocular lens implant at age 52 years and a right cataract was diagnosed at age 56 years. Physical examination did not reveal cutaneous tumours or CALs. A mild right hemiparesis was present.

Ocular examination revealed corrected visual acuities of 20/25 in both eyes. She had a unilateral VII nerve weakness with poor lid closure. She had normal fields, colour vision and extraocular move- ments, absent corneal sensation in both eyes and a mild left ptosis due to the gold weight implant in the upper lid. There were no iris hamartomas. She was pseudophakic in the right eye and had mild spokelike cortical lens opacities in the left eye. Fundus examination showed a pallorous optic disc on the left eye and a tilted optic disc on the right with a peripapillary astrocytic hamartoma (Fig. 3). Multiple, subtle, small hypopigmented areas in the choroidal layer were scattered throughout both fundi.

\section{Case 4}

This 25-year-old man was diagnosed with NF2 following an MRI scan that revealed bilateral VSs and cervical tumours. Since age 12 years he had had blurring of vision in his left eye and horizontal and vertical diplopia on left gaze. For several years prior to presentation he had suffered from headaches, and mild loss of hearing in the right ear, and for the previous 2 years, tinnitus and unsteady gait. He had a small subcutaneous tumour, histology unknown, removed from his left ear. There was no family history of NF2. The general physical examination was normal.

Ocular examination showed visual acuities of $20 / 25$ unaided in the right eye and 20/40 with -1.25 / $+0.50 \times 30^{\circ}$ in the left eye. There was a small tumour on the left upper lid. He had a left hypertropia of 20 prism dioptres and exotropia of 12 prism dioptres in primary position. His extraocular movements revealed a pupil-involving left inferior branch oculomotor palsy. He had no nystagmus. Corneal sensation was reduced on both sides. There were no iris 


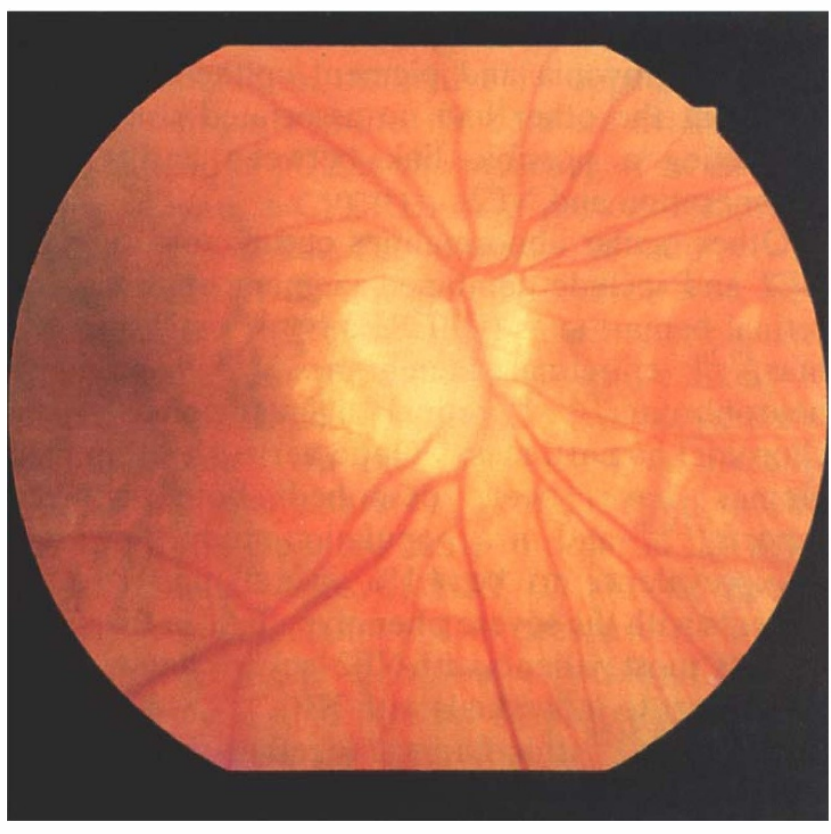

(a)

hamartomas. He had a mild posterior capsular lens opacity on the right and a moderate posterior capsular plaque-like lens opacity on the left (Fig. 4). He had a series of flat retinal holes with lattice degeneration in the periphery of both retinae, with a small operculated tear with a surrounding cuff of subretinal fluid superiorly in the left eye. There was no posterior vitreous detachment.

\section{Case 5}

This 37-year-old man had a history of progressive right ptosis for 15 years, associated with progressive

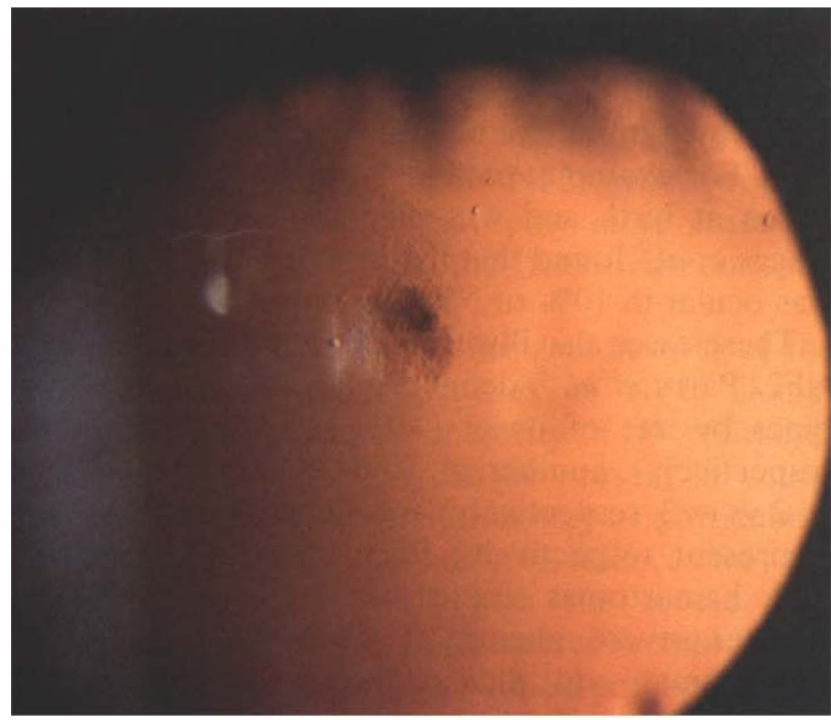

(a)

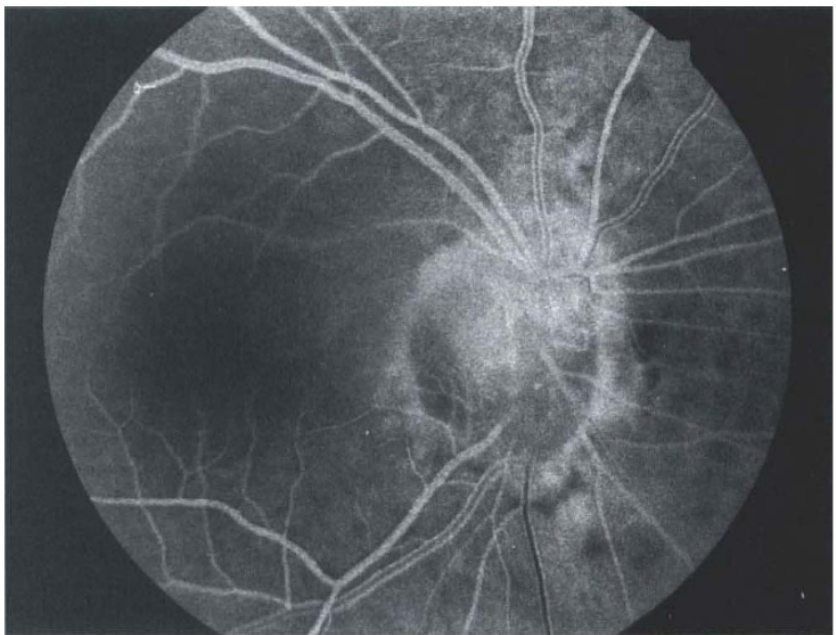

(b)

Fig. 3. Case 3. Colour (a) and fluorescein angiogram appearance $(b)$ of the right fundus, showing mild masking of underlying fluorescence by the peripapillary hamartoma (arrows). (Detail published in Ragge et al. ${ }^{7}$ )

downward deviation of the right eye over the previous 4 years. He had also noticed clouding of his vision more on the right side than the left for the previous 2 years. He had a spinal tumour operated on at age 7 years. He developed progressive hearing loss bilaterally at age 10 years from bilateral VSs. Three operations for removal of left VSs were performed at ages 24, 28 and 32 years. A right VS was also removed, but subsequently recurred. $\mathrm{He}$ had a left posterior fossa meningioma resected at age 30 years, a right posterior fossa meningioma resected at age 34 years and a left parasagittal meningioma

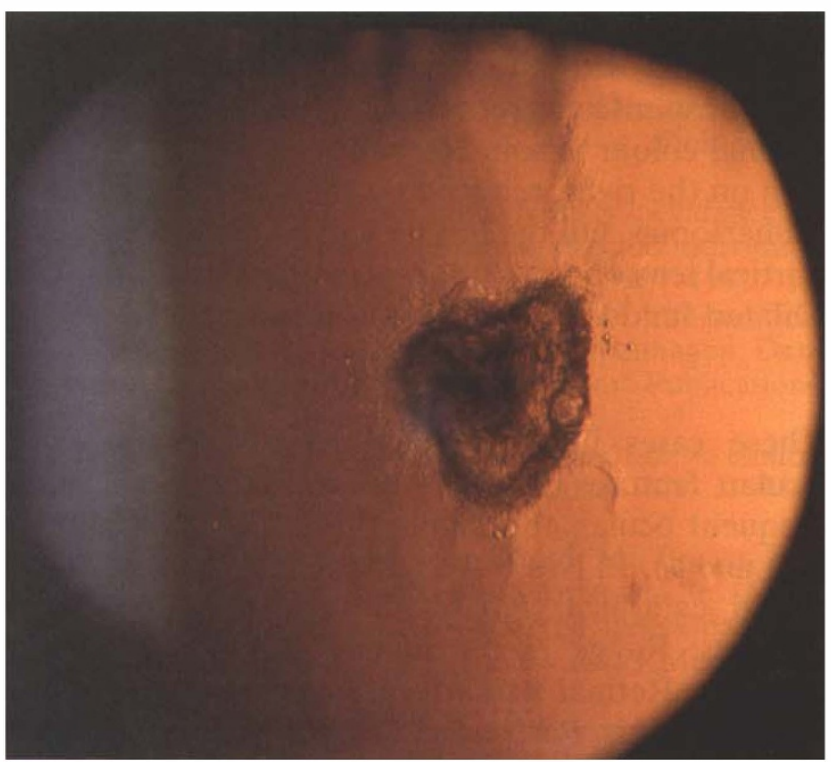

(b)

Fig. 4. Slit lamp views of cataracts showing early posterior subcapsular lens opacity in the right eye (a) and moderate posterior subcapsular lens opacity in the left eye $(b)$. 


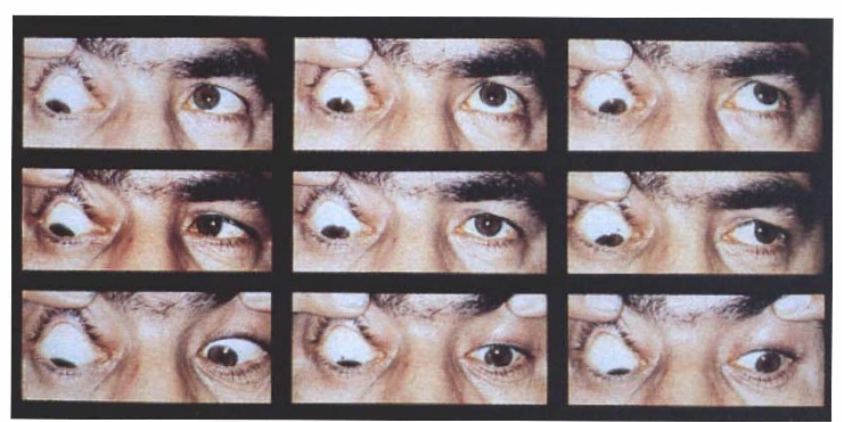

Fig. 5. Case 5. The nine positions of gaze demonstrating downward deviation of the right eye and right superior division III nerve palsy.

resected 1 year later. He had a tumour removed from his skull vertex at age 35 years and back surgery for tumours at ages 33 and 36 years. Other lesions present on CT scan included extensive falx meningioma, cavernous sinus meningioma, recurrent right VS, C3 and C5 spinal canal tumours, a left brachial plexus mass, a pseudoaneurysm of the left axillary artery, and paraspinal masses in the high cervical level and at T10. Physical examination showed five cutaneous tumours, spinal scoliosis, bilateral VII nerve paresis, cerebellar dysfunction and a sensorimotor neuropathy of C7. His father became deaf for unknown reasons at age 38 years and died at age 44 years from a vascular event. His mother was well at age 56 years. He has two brothers with hearing difficulties and one healthy brother.

Ocular examination showed corrected visual acuities of right eye $20 / 70$ with $-1.00 /+1.00 \times 10^{\circ}$, left eye $20 / 40$ with $+1.00 /+0.50 \times 10$. He had a total right ptosis, with right hypotropia. Extraocular movements were normal on the left, but severely restricted on the right with inability to elevate the right eye to primary position, implying a superior division of III nerve palsy with inferior rectus fibrosis (Fig. 5). His pupil responses were normal. He had full fields and normal colour vision. He had absent corneal sensation on the right, reduced on the left. He had no iris hamartomas. On the left he had an off-axis posterior cortical lens opacity, but no lens opacity on the righ. Dilated fundus examination was normal.

\section{DISCUSSION}

These cases illustrate many of the characteristic ocular features of NF2. Cataracts are the most frequent ocular abnormality (e.g. PSCC in patients 1,2 and 4). In two large patient series, Parry et al. ${ }^{10}$ found cataracts (PSCC and cortical) in $81 \%$ of patients; Ragge et al. ${ }^{7}$ found cataracts in $69 \%$ of patients. Retinal hamartomas occur less frequently than cataracts. Parry et al. ${ }^{10}$ and Ragge et al..$^{7}$ found retinal hamartomas in $8 \%$ and $22 \%$ of patients, respectively. In the latter series, juvenile onset ocular motor abnormalities, as seen in patients 1,4 and 5, affected $12 \%$ of NF2 patients..$^{15}$ Two of the cases reported here had retinal tears; one was associated with high myopia and pigment epithelial abnormality, but the other had no associated risk factors, suggesting a possible link between early retinal degeneration and NF2.

Other ocular abnormalities occur more rarely in NF2 and include combined pigment epithelial and retinal hamartomas (CPERH), ${ }^{7.16-18}$ optic disc gliomas, ${ }^{19,20}$ epiretinal membranes, ${ }^{5.7 .20}$ conjunctival neurofibroma, ${ }^{7,21,22}$ retinal haemangiomas, ${ }^{23}$ and choroidal hamartomas. ${ }^{24}$ Optic nerve sheath meningiomas have been described both in case reports $5,25.26$ and in a population study that found the prevalence to be $4.1 \%$ overall and $8.6 \%$ in patients with the severe phenotype. ${ }^{9}$ As in these case reports, most patients with NF2 lack iris hamartomas, in contrast to individuals with NF1. ${ }^{4.27}$ Although not absolute, ${ }^{5,7,24,28}$ this finding, together with the presence of cataract, provides an important diagnostic tool for differentiating NF2 from NF1. ${ }^{15}$

Presymptomatic diagnosis of NF2 is possible using DNA markers and mutation analysis. ${ }^{29.30}$ However, linked DNA markers can only be used in at-risk members of multigeneration families, and mutation analysis cannot detect all mutations. Therefore, clinical signs and symptoms will remain important in diagnosis, particularly for sporadic cases. Parry et $a l .{ }^{10}$ found that the initial manifestation of NF2 in $44.4 \%$ of patients was related to VSs (hearing loss, tinnitus plus hearing loss, imbalance); $22.2 \%$ had symptoms related to other CNS tumours; $12.7 \%$ had vision loss or diplopia; and $7.9 \%$ were asymptomatic. While ocular symptoms were the initial manifestation in a minority of cases, it is important to note that the mean age of onset of ocular symptoms was 5.6 years, compared with 14.1, 20.6 and 25.7 years for symptoms related to skin abnormalities, other CNS tumours and VS, respectively. Thus, attention to ocular symptoms can be crucial in early detection of NF2. For example, patient 2 in this study had poor vision at birth and was diagnosed at age 3 years. Ragge et $\mathrm{l}^{7}{ }^{7}$ found that the first presenting symptom was ocular in $10 \%$ of NF2 patients.

These cases also illustrate phenotypic variability in NF2. Parry et al. ${ }^{10}$ defined mild and severe phenotypes by age of onset $(\geqslant 20$ years vs $<20$ years, respectively), number of other intracranial tumours $(<2$ vs $\geqslant 2$, respectively) and spinal tumours (absent vs present, respectively). Parry et al. ${ }^{10}$ also proposed that hamartomas could be used to distinguish another severe phenotype. Patients 1 and 2 had hamartomas and did have a severe phenotype. However, patient 3 had a mild phenotype including diagnosis at age 28 years, one other intracranial tumour, no spinal tumours and a slow clinical course. Thus, the presence of hamartomas is not an absolute correlate of severe phenotype in NF2. 
Many of the ocular findings in NF2 are rare in the general population, distinctive in appearance and often present early in life. No data are available for the likelihood of NF2 in the presence of an isolated finding of, say, a CPERH, or a posterior capsular cataract in a child. The majority of adults with isolated CPERH do not have NF2. However, NF2 patients with CPERH are often new mutations and have more severe disease and are more likely to present in childhood (D. R. G. Evans, personal communication). On the other hand an isolated posterior capsular cataract in a child is a less specific sign and would be more likely to occur in the more typical, familial cases of NF2 (D. R. G. Evans, personal communication). The presence of any of these unusual ocular signs, but especially CPERH, should arouse a high level of suspicion and should prompt a search for neurological, auditory and dermatological signs, and an inquiry into family history. If any of these are positive, this would be highly suggestive of NF2 and screening for VSs needs to be considered. If all are negative, it would be prudent to follow these individuals, at least on a yearly basis, in a paediatric neurology or genetics clinic until a time when they could be considered negative for NF2. Clearly once a highly sensitive genetic test becomes available, many of the uncertainties concerning screening will disappear and resources can be concentrated on those individuals with the disease.

In addition to retinoblastoma, ocular abnormalities are found in several tumour suppressor gene syndromes such as NF1, ${ }^{15}$ von Hippel-Lindau disease $^{31}$ and familial adenomatous polyposis coli. ${ }^{32}$ However, the neurofibromatoses are especially noteworthy given the presence of developmental ocular abnormalities in addition to the tumours. The NF1 gene product, neurofibromin, may have important roles in various tissues at different developmental stages. ${ }^{33}$ The NF2 gene product, schwannomin or merlin, may play more extended roles in development of the lens, vitreous and retina.

In summary, these cases illustrate several of the most important ocular features of NF2. Ocular abnormalities occur in the majority of NF2 patients, and because ocular signs can be present in young children, the ocular examination may be particularly important in early diagnosis. Evidence for the association of hamartoma with the severe NF2 phenotype is suggestive, but requires further definition. The molecular and biochemical pathogenesis of ocular abnormalities in NF2 is unknown and requires elucidation. The developmental nature of the ocular changes suggests that the NF2 gene may play a role not only in growth regulation, but also in ocular development.
Presented at the Annual Congress of Ophthalmologists, Guernsey, 1994. The cases presented in detail here were included in a large survey published in Am J Ophthalmol 1995;120:634-41.

We thank R. Shannon, PhD, R. Nelson, MD, Jana Klein, MS, and the California NF2 Research Group for their support; A. Linn Murphree, MD, Mark Borchert, MD, and the Pediatric Ophthalmology group at Childrens Hospital Los Angeles for their help and providing facilities; and Ms W. Macnamara for photographic assistance. This study was supported by the Vista Foundation, Los Angeles, California; House Ear Institute grant 484; and the Neurofibromatosis Association, Kingston-upon-Thames, United Kingdom.

Key words: Astrocytic hamartoma, Cataract, Neurofibromatosis 2, Eye, Epiretinal membrane, Tumour suppressor gene, Combined pigment epithelial and retinal hamartoma.

\section{REFERENCES}

1. Rouleau GA, Merel P, Lutchman M, et al. Alteration in a new gene encoding a putative membrane-organizing protein causes neurofibromatosis type 2. Nature 1993;363:515-21.

2. Trofatter JA, MacCollin MM, Rutter JL, et al. A novel moesin-, ezrin-, radixin-like gene is a candidate for the neurofibromatosis 2 tumor suppressor. Cell 1993;72: 791-800.

3. Sainz J, Huynh DP, Figueroa K, Ragge NK, Baser ME, Pulst SM. Mutations of the neurofibromatosis type 2 gene and lack of the gene product in vestibular schwannomas. Hum Mol Genet 1994;3:885-91.

4. Kaiser-Kupfer M, Friedlin V, Datiles M, et al. The association of posterior capsular lens opacities with bilateral acoustic neuromas in patients with neurofibromatosis type 2. Arch Ophthalmol 1989;107:541-5.

5. Kaye L, Rothner A, Beauchamp G, Meyers S, Estes M. Ocular findings associated with neurofibromatosis type 2. Ophthalmology 1992;99:1424-9.

6. Bouzas EA, Freidlin V, Parry DM, Eldridge R, KaiserKupfer MI. Lens opacities in neurofibromatosis 2: further significant correlations. $\mathrm{Br} \mathrm{J}$ Ophthalmol 1993;77:354-7.

7. Ragge NK, Baser ME, Klein J, et al. Ocular abnormalities in neurofibromatosis 2. Am J Ophthalmol 1995;120:634-41.

8. Eldridge R, Parry D. Neurofibromatosis 2: evidence for clinical heterogeneity based on 54 affected individuals studied by MRI with gadolinium, 1987-1991. In: Conference Proceedings of the First International Conference on Acoustic Neuroma, Copenhagen, Denmark. Amsterdam/New York: Kugler Publications, 1991.

9. Evans DRG, Huson SM, Donnai D, et al. A clinical study of type 2 neurofibromatosis. Q J Med 1992;84:603-18.

10. Parry DM, Eldridge R, Kaiser-Kupfer MI, Bouzas EA, Pikus A, Patronas N. Neurofibromatosis 2 (NF2): clinical characteristics of 63 affected individuals and clinical evidence for heterogeneity. Am J Med Genet 1994:52:450-61.

11. Merel P, Hoang-Xuan K. Sanson M, et al. Screening for germ-line mutations in the NF2 gene. Genes Chrom Cancer 1995;12:117-27.

12. Wertelecki W, Rouleau GA, Superneau DW, et al. Neurofibromatosis 2: clinical and DNA linkage studies of a large kindred. N Engl J Med 1988;319:278-83. 
13. Bourn D, Carter SA, Mason S, Evans DGR, Strachan T. Germ-line mutations in the neurofibromatosis 2 gene in multiple tumor types. Hum Mol Genet 1994;3:813-6.

14. Consensus Development Panel National Institutes of Health Consensus Development Conference Statement on Acoustic Neuroma, 11-13 December 1991. Arch Neurol 1994;51:201-7.

15. Ragge NK. Clinical and genetic patterns of neurofibromatosis 1 and 2. Br J Ophthalmol 1993;77:662-72.

16. Landau K, Dossetor FM, Hoyt, WF, Muci-Mendoza R. Retinal hamartoma in neurofibromatosis type 2. Arch Ophthalmol 1990;108:328-9.

17. Good W, Brodsky M, Edwards M, Hoyt W. Bilateral retinal hamartomas in neurofibromatosis type $2 . \mathrm{Br} \mathrm{J}$ Ophthalmol 1991;75:190.

18. Bouzas EA, Parry DM, Eldridge R, Kaiser-Kupfer MI. Familial occurrence of combined pigment epithelial and retinal hamartomas associated with neurofibromatosis 2. Retina 1992;12:103-7.

19. Dossetor FM, Landau K, Hoyt WF. Optic disk glioma in neurofibromatosis type 2. Am J Ophthalmol 1989;108:602-3.

20. Landau K, Yasargil GM. Ocular fundus in neurofibromatosis type 2. Br J Ophthalmol 1993;77:646-9.

21. Kalina PH, Bartley GB, Campbell RJ, Buettner H. Isolated neurofibromas of the conjunctiva. Am J Ophthalmol 1991;111:694-8.

22. Perry $\mathrm{H}$. Isolated neurofibromas of the conjunctiva. Am J Ophthalmol 1992;89:112-3.

23. Frenkel M. Retinal angiomatosis in a patient with neurofibromatosis. Am J Ophthalmol 1967;63:804-8.

24. Garretto NS, Ameriso S, Molina HA, et al. Type 2 neurofibromatosis with Lisch nodules. Neurofibromatosis $1989 ; 2: 315-21$.
25. Shapland CD, Greenfield JG. A case of neurofibromatosis with meningeal tumour involving the left optic nerve. Trans Ophthalmol Soc UK 1935;55:257-79.

26. Cunliffe IA, Moffat DA, Hardy DG, Moore AT. Bilateral optic nerve sheath meningiomas in a patient with neurofibromatosis type 2 . $\mathrm{Br} \mathrm{J}$ Ophthalmol 1992;76:310-2.

27. Pearson-Webb M, Kaiser-Kupfer M, Eldridge R. Eye findings in bilateral acoustic (central) neurofibromatosis: association with presenile lens opacities and cataracts but absence of Lisch nodules. N Engl J Med 1986;315:1553-4.

28. Charles SJ, Moore AT, Yates JRW, Ferguson-Smith MA. Lisch nodules in neurofibromatosis type 2. Arch Ophthalmol 1989;107:1571-2.

29. MacCollin M, Mohney T, Trofatter J, Wertelecki W, Ramesh V, Gusella J. DNA diagnosis of neurofibromatosis 2: altered coding sequence of the merlin tumor supressor in an extended pedigree. JAMA 1993;270:2316-20.

30. Ruttledge MH, Narod SD, Dumanski JP, et al. Presymptomatic diagnosis for neurofibromatosis 2 with chromosome 22 markers. Neurology 1993; 43:1753-60.

31. Moore AT, Maher ER, Rosen P, Gregor Z, Bird AC. Ophthalmological screening for von Hippel-Lindau disease. Eye 1991;5:723-8.

32. Olschwang S, Tiret A, Laurent-Pulg P, Muleris M, Parc $\mathrm{R}$, Thomas $\mathrm{G}$. Restriction of ocular fundus lesions to a specific group of APC mutations in adenomatous polyposis coli patients. Cell 1993;75:959-68.

33. Danglot G, Regnier V, Fauvet D, Vassal G, Kujas M, Bernheim A. Neurofibromatosis 1 (NF1) mRNAs expressed in the central nervous system are differentially spliced in the 5 ' part of the gene. Hum Mol Genet 1995;4:915-20. 